\title{
INVESTIGATION ON THE PRINTABILITY OF SPECIALTY PAPERS FOR LUXURY LABELS
}

\author{
Dimitrina Todorova $^{1}$ (D), Nevena Pavlova ${ }^{2}$ \\ ${ }^{1}$ University of Chemical Technology and Metallurgy, Department of Pulp, Paper and \\ Printing art, Sofia, Bulgaria \\ ${ }^{2}$ Rotoprint, Industrialna zona, Ravno pole, Bulgaria
}

\begin{abstract}
Specialty luxury label papers mainly are results from the search for all that stimulates our creativity and senses. They are from papers that awaken the desire to create, as a magnificent mix of texture and colours that elevates luxury packaging to the category of art and a brilliant collection that ultimately shows that luxury packaging really is. In order to achieve the desired complex effect on the customer with its combined impact through the texture, colour of the paper and graphic design for each manufacturer of luxury labels, it is undoubtedly essential to be familiar with the printability of the paper used.

The aim of the present work was to study the main properties of the commonly used papers for printing of self-adhesive labels, in view of their printability. It is especially important for the production, as well as for the awareness of the designers working in this field, to know the specifics of these papers, to know the main indicators influencing their printability, to know all the facts allowing the right choice of printing technology and finishing processes. To achieve this goal, various properties of five types of paper have been determined: structural-dimensional, physical-mechanical, capillary-hygroscopic and optical. In addition, with all paper analysis, a printing sample have been made with different types of printing (offset UV, flexo UV, screen, cold foil, hot foil, silk foil) and converting (emboss, laser cutting, laser engraving) techniques. Based on the obtained results, new designer labels for wine have been printed. The results show that there is a clear dependence between the paper composition, its main and specific properties and the quality of the different printing and finishing processes, as well as the overall appearance of the produced labels. The printing and finishing processes have been carried out in Rotoprint Printing House, Bulgaria and the obtained results could give a valuable information not only for the particular printing house, but for other specialists in the field of labels production.
\end{abstract}

Key words: specialty papers, printability, self-adhesive labels, strength properties, printing, converting

\section{INTRODUCTION}

Predecessors of modern labels are the brands that the producers of beverages (mainly wines) put on amphora, bottles and other clay dishes. The advent of paper in Europe has had a major impact on the appearance of labels. By 1820, wine labels began to resemble those that exist today, but are characterized by a strict and compact style. Initially, they have a simple rectangular or oval shape. Over the time, all kinds of ornaments and coats of arms, angels, saints, monks, folk scenes and picturesque landscapes, etc. enter the production of labels. In those days there were no official rules for the content of labels: they usually contained only information on the geographical origin, the producer and the place of production. In 1834, winemakers began to put on labels and information about the year of harvest. For the period from 1820 to 1920, the production of champagne increased from 2 to 20 million bottles per year, and last but not least, the label played a big role in its 10-fold increase. The small piece of paper becomes a powerful engine of trade and a means of information for customers. The first official rules for labeling wines appeared in the early 20th century. Since then, more and more attention has been paid to labels: such famous artists as Salvador Dali, Marc Chagall, Pablo Picasso and Andy Warhol are working on their design. The pioneer in this field is the Bordeaux area, with the famous example of Mouton-Rothschild, which in 1924 started to ask an artist to create the label for each vintage of its wine (Obis, 2018; Franson, 2006).

Paper is almost as old as communication itself. Plus, it is as versatile and technologically advanced as any electronic chip. Most of the paper producers work hard to provide high quality and value-added papers, with striking surfaces and textures, vivid colours and papers, that can be printed in every possible way. Label papers are highly functional papers, that can catch our eye or remain discreetly in the background. Luxury label papers are not just a means of communication - they are simply art in the field of: olive oils, red, white and sparkling wines, spirits and liqueurs, gourmet, beers and others (Franson, 2006; UPM Raflatac, n.d.; EUR-Lex, 2015; Labels \& Labeling, 2010). 
Labels for packaged products can come in a variety of sizes and shapes. They range from those without an adhesive layer, which are glued with glue, In-mold labelling - IML (which are built into the package when casting it) to self-adhesive, which can be sensitive to pressure, heat (self-adhesive thermocouple).

Self-adhesive labels are made on the basis of paper and synthetic film coatings. Surface coating is applied to improve the properties of the label paper. Modern technologies allow the production of surface-coated paper using a base, both from cellulose and with the participation of secondary fibrous material. The use of surface-coated paper allows to significantly increase the quality of the print and the artistic layout. The advantage of surface-coated paper is the smooth flat surface, which has better printing properties than the uncoated one. As a result of different calendaring techniques, the paper acquires gloss or matt. Excessive gloss is not always desirable, as the matte surface is better for reading. Of particular importance for surfaceenhanced printing paper is its "dusting and lintting" resistance.

For the production of labels, one-sided coated paper is used, suitable for further processing, which must be resistant to twisting and moisture. Coated papers should contain an increased amount of binder to prevent ink from passing through. With continuous application of printing inks on the paper, no additional measures are required to ensure varnish impermeability, because they themselves give the paper the necessary barrier properties. Among the advantages of surface-coated paper is its density and the high degree of surface uniformity in the absorption of printing inks. The additional requirements for this type of paper in terms of hydrophobicity are due to the wetting of its surface during the offset printing process and the end-user's application.

Self-adhesive labels refer to adhesive materials, the adhesive ability of which does not require activation by wetting or temperature exposure. The gluing of this type of labels is carried out by joining them to heterogeneous surfaces and their subsequent pressing or smoothing. They are most common in:

- labels for packaging of various goods;

- $\quad$ sticking to food prices;

- $\quad$ self-adhesive labels on electronic scales;

- $\quad$ high quality full colour advertising production on white, coloured, fluorescent, metallized paper;

- labels of wine and alcoholic beverages;

- $\quad$ postage stamps, envelopes, folders, cassettes;

- $\quad$ transport and production marking for different purposes.

The properties of the paper depend on the properties of the source fibrous materials, their morphological structure and chemical composition, the degree and nature of grinding, the presence of fillers, sizing agents and other chemical additives, as well as the technological conditions of production.

Each type of paper is characterized by a certain combination and level of quality indicators, due to the specificity of the conditions in which it is used. The variety of areas of application of the paper determines the diversity in the properties required by it (Holik, 2006; Thorn and On Au, 2009).

The aim of the present work is to study the main properties of the commonly used papers for printing of self-adhesive labels, in view of their printability. It is especially important for the production, as well as for the awareness of the designers working in this field, to know the specifics of these papers, to know the main indicators influencing their printability, to know all the facts allowing the right choice of printing technology and finishing processes. To achieve this goal, various properties of five types of paper have been determined: structural-dimensional, physical-mechanical, capillary-hygroscopic and optical. In addition, with all paper analysis, a printing sample have been made with different types of printing (offset UV, flexo UV, screen, cold foil, hot foil, silk foil) and converting (emboss, laser die-cutting, laser engraving) techniques. Based on the obtained results, new designer labels for wine have been printed.

\section{MATERIALS AND METHODS}

Self-adhesive wine label papers are a special type of papers characterized by a variety of fiber compositions, chemical additives, production technology and properties. Due to this feature, very often the product characteristics of the papers from different manufacturers are different, incomplete, insufficient or completely missing. Therefore, the complete characterization of the papers used in the printing house is essential for the optimization of the production processes and leads to the successful realization of the produced products. In the present work five types of papers for self-adhesive luxury wine labels have been used: Pure Cotton, Symphonie, Blizzard, Organdie and Velin Caviar, delivered from Rotoprint printing house. 


\subsection{Microscopic analyses}

The microscopic analyses of cellulose and paper materials, is a specific analysis generally used to determine the fiber composition of the paper and to study the structure and size of the source fibers. Small amount of test fibers (pre-milled mechanically) is placed in a porcelain pounder with a few drops of $1 \% \mathrm{NaOH}$. This is followed by rinsing the sample several times with distilled water on a fine metal mesh. Three samples of fibers have to be placed on a glass slide, distribute well with the help of pins, and remove excess water with the help of filter paper. A drop of Herzberg's reagent (Cl - Zn - J) (ISO 9184-3:1990) is dropped on each of the samples and again very carefully the fibers should be distributed. Finally, the samples are dried at about $60^{\circ} \mathrm{C}$ with a lamp. After cooling down to room temperature, each sample is covered with a thin glass slide, so the fiber samples to be evenly distributed, free of accumulations and air bubbles. Stained fiber samples are ready to be observed under a microscope at different magnifications (Ivanova et al, 2009).

To determine the type of fibers, the peculiarities of their structure are studied - shape and size (whether they are flat or cylindrical); presence of tubules - size and shape; appearance of their ends; size, type and location of the pores, as well as the size and shape of the cells. The colour and morphological features of the fibers determine the type of raw material from which the paper sample is obtained. The Herzberg reagent stains the fibrous materials in the following ways (Ivanova et al, 2009): Cotton, linen and hemp pale to intense pink - red; Cellulose - blue to violet colour, while the sulphite cellulose is coloured more intensely, and the sulphate - paler and darker with brownish hues; Wood pulp and semi-cellulose - from light yellow to yellow-brown colour depending on the yield; Wool - pale yellow colour; Artificial fibers - blue colour; Acetate fibers - yellow colour; Synthetic fibers - with no colour.

For the microscopic characterization of the natural fibers according to their morphological features, the following three characteristics are examined: general appearance of the fibers (size and configuration); fiber marking (type, size and location of pores, presence of channel, nature of the ends), accompanying structural elements (core rays, cells and vessels).

\subsection{Paper Properties}

According to ISO 187: 1990, the conditioning and subsequent testing of paper samples was performed under the following conditions: Relative humidity - $50 \pm 2 \%$; Temperature $-23 \pm 1{ }^{\circ} \mathrm{C}$, the required time for conditioning the paper before the test is at least $4 \mathrm{~h}$.

Studies have been conducted to determine:

- Composition - type of fibrous materials;

- Structural and dimensional properties - basic weight - ISO 536: 1998; thickness - ISO 534: 2006; density - ISO 534: 2006; porosity; Bekk smoothness (ISO 5627 / A1: 2004);

- Hygroscopic properties - water absorption Cobb60 (ISO 535:2014);

- Strength properties - tensile index - ISO 1924-2: 2000; burst index - ISO 2758: 2005, tear index ISO 1974:2012

- Optical properties - whiteness CIE, whiteness CIE - UV, ISO brightness, ISO brightness - UV, fluorescense CIE, fluorescense ISO brightness (ISO 2470: 2016); colour coordinates - L*, a*, b* (ISO 5631-3:2008).

\subsection{Printing of samples on the tested papers}

Printed sample, presented in Figure 1, was applied to all five tested types of paper. The figure shows the main types of printing and converting processes in the production of self-adhesive labels, in the conditions of Rotoprint printing house. The used printing and converting machines are:

Gallus EM 280 - UV flexographic printing and rotary screen printing, hot foil embossing and cold foil printing. Possibility to print in 8 colours; width of the material from $120 \mathrm{~mm}$ to $270 \mathrm{~mm}$; maximum size of the printed product $440 \mathrm{~mm} \times 250 \mathrm{~mm}$; average printing speed about $80 \mathrm{~m} / \mathrm{min}$.

Gallus TCS 250 - combines a wide range of printing, processing and finishing options. Modular seven offset press including finishing processes such as screen printing, hot foil embossing, varnishing and relief embossing, lamination section, flexo section for complete and partial varnishing, embossing, 3D embossing and flat punching. Printing with UV offset inks and varnishes with maximum format of the printed product $254 \mathrm{~mm} \times 254 \mathrm{~mm}$; maximum paper width $260 \mathrm{~mm}$; average machine speed 7500 stroke/hour; suitable for short runs with complex designs requiring the use of special types of paper and perfect quality.

Cartes GT 364 HSDL - machine for finishing operations with section for flat hot stamping, printing of two different foils at the same time, section for flat screen, Silk foil, embossing, flat bed die-cutting, laser die- 
cutting, different types of perforations, engraving, perforation of individual sections, numbering and custom information, disc knives for splitting and trimming the material with average machine speed 5000 stroke/hour; maximum width of the material $360 \mathrm{~mm}$; maximum area of the printed field $250 \mathrm{~mm} \times$ $360 \mathrm{~mm}$.

The printability of each paper sample was visually evaluated on the basis of the reproduced printed elements. In Rotoprint to maintain an excellent print quality a spectrophotometric calibration is done periodically (on every six months) by which a calibration curves are constructed for each sample and the results are embedded in the prepress software.

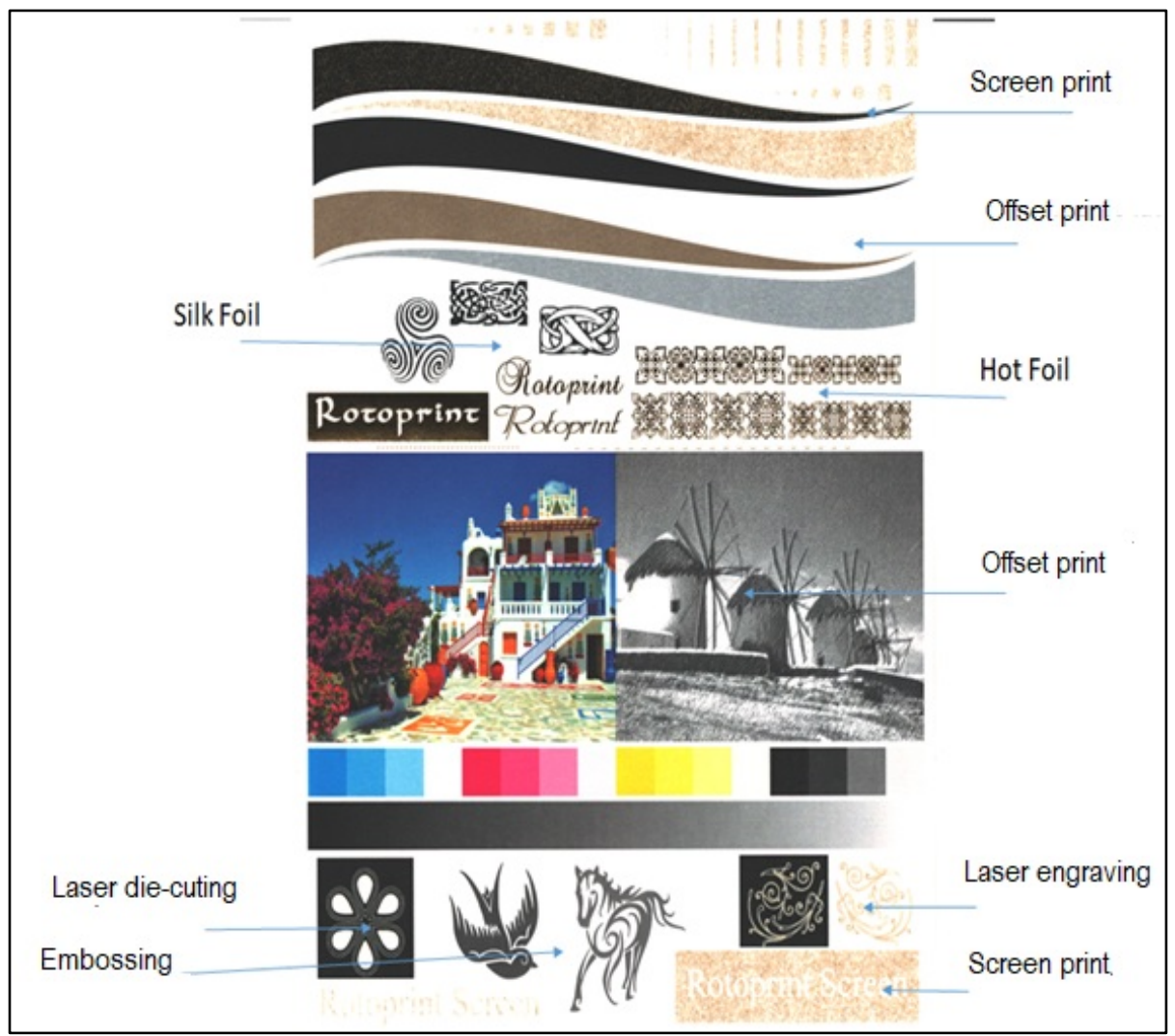

Figure 1: Printed sample

\section{RESULTS AND DISCUSSIONS}

For the extensive characterization of the studied five types of papers for self-adhesive labels, a variety of laboratory analyzes were performed followed by printing samples, on the basis of which the connection between the properties of the papers and their printability was considered. Based on the conclusions of the research, new designer wine labels have been made, which have been printed and processed using various printing and finishing techniques.

\subsection{Investigation on the fiber content of the papers}

Figure 2 shows the microscopic analysis of Pure Cotton paper. It is visible that this type of paper contains $100 \%$ natural cotton fibers, which under the "Cl-Zn-J" indicator are stained in pale to intense pink-red colour and have the typical natural cotton morphology: long fibers, tape-shaped, without pointed edges with thin walls and in the center passes a wide channel (up to 2/3 of the width), with no pores and thickenings. 


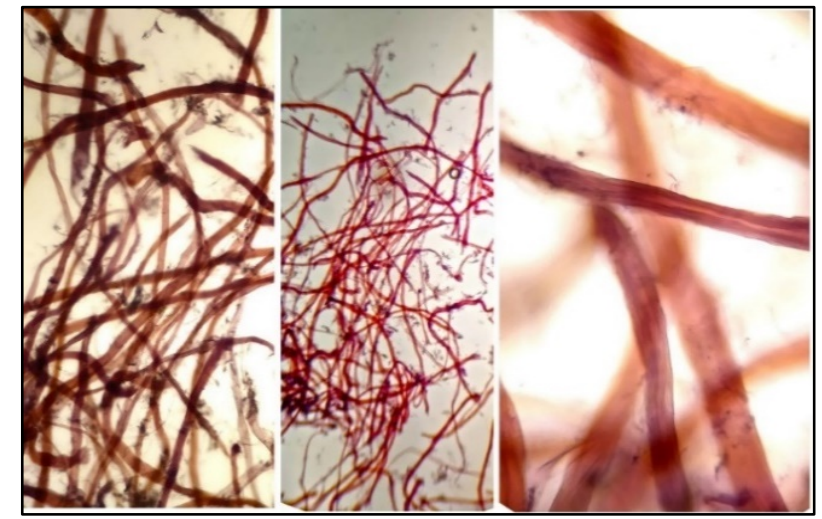

Figure 2: Microscopic analysis of Pure Cotton paper

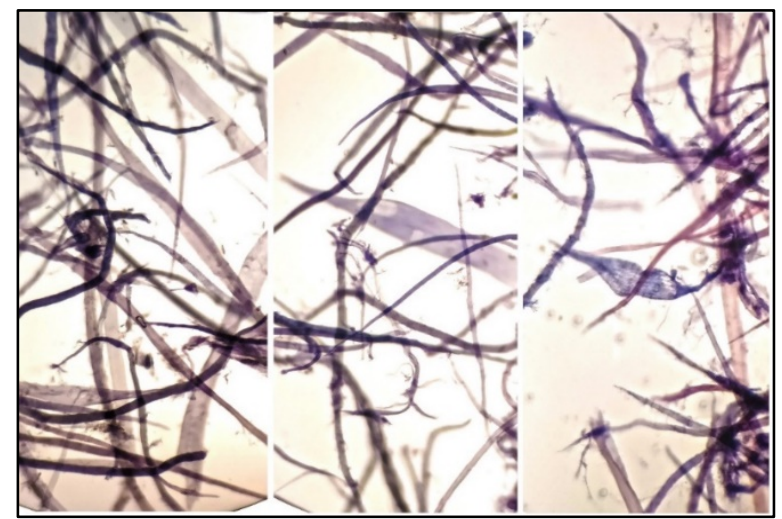

Figure 3: Microscopic analysis of Symphonie paper

The results of the microscopic analysis of Symphonie paper are presented in Figure 3. The fibers of this type of paper have the typical for bleached fibrous materials, of coniferous and deciduous wood species, blue to violet colouring under "Cl-Zn-J" indicator. Therefore, the paper consists of $100 \%$ bleached cellulose with a lignin content less than $9 \%$. Regarding the specific tree species, the figure shows that the Symphonie paper contains of bleached softwood cellulose from spruce wood and bleached hardwood cellulose from poplar wood.

The composition of Blizzard paper is multicomponent, which is clearly outlined by the heterogeneous picture presented in Figure 4. This paper consists of not more than 5\% natural cotton fibers, softwood cellulose from spruce wood and two types of hardwood cellulose - from poplar and beech wood. Predominant in the composition of the paper is the cellulose from poplar wood, in the range of $50 \%$.

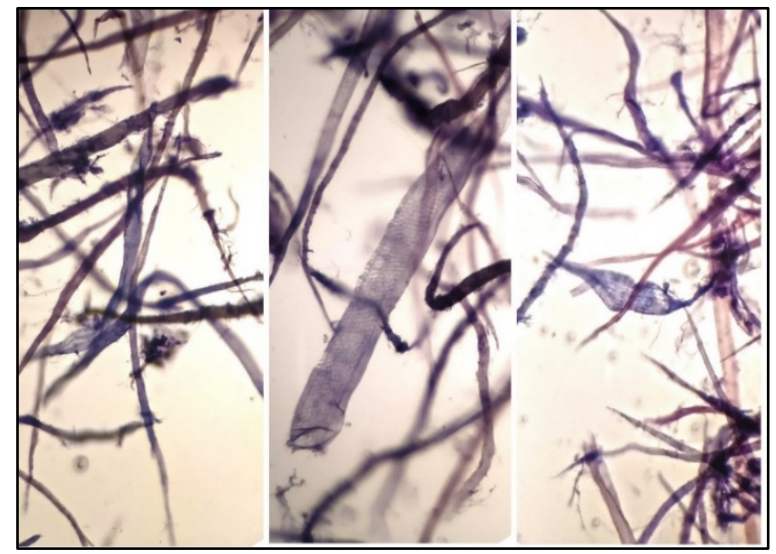

Figure 4: Microscopic analysis of Blizzard paper 


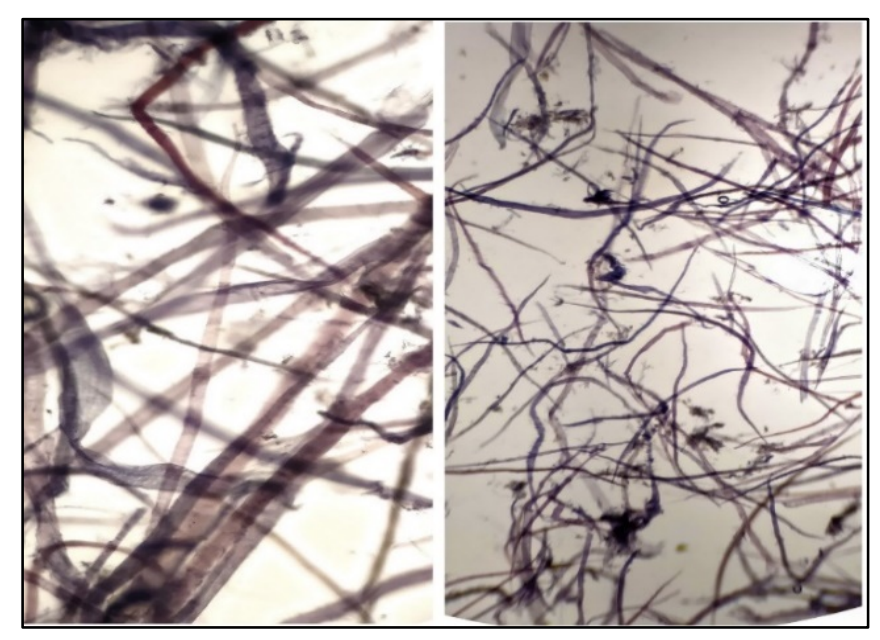

Figure 5: Microscopic analysis of Organdie paper

The microscopic analysis of the fourth type of paper - Organdie is presented in Figure 5. This paper consists of small amount of natural cotton fibers, not more than $10 \%$ and bleached softwood cellulose from spruce wood and bleached hardwood cellulose from beech wood, with the predominant cellulose content of the coniferous specie.

Figure 6 shows the microscopic analysis of Velin Caviar paper. This is the paper with the largest variety of types of cellulose fibrous materials used. It consists of about $10 \%$ natural cotton cellulose, $40 \%$ softwood cellulose of two wood types - spruce and pine, in a ratio of 1:1 and hardwood cellulose from two species of wood - poplar and beech wood, again in a ratio of 1:1, with a total content of $50 \%$.

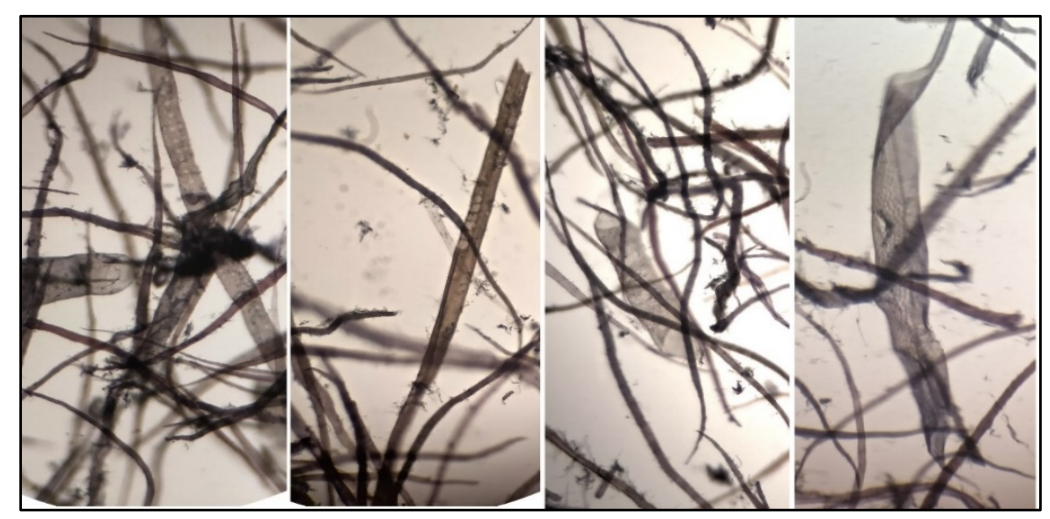

Figure 6: Microscopic analysis of Velin Caviar paper

\subsection{Paper properties investigation}

The results from the analysis of the investigated structural and dimensional properties, hygroscopic, strength and optical properties of the studied papers are presented in Table 1.

The investigated papers are with a basic weight in the range of $90-100 \mathrm{~g} / \mathrm{m}^{2}$ and thickness between 0,09 - $016 \mathrm{~mm}$, but most interesting are the results for the density and porosity of the papers, because they have bigger deviation which means that even at similar basic weight papers probably will have different ability of emboss and engraving during the converting processes. The smoothness of the paper is the most important characteristic of the paper for the engraving printing, which means that most suitable could be Blizzard paper. From the Cobb analysis is seen that Blizzard paper has the optimal for the offset printing Cobb values $\sim 22 \mathrm{~g} / \mathrm{m}^{2}$ and we could expect excellent offset printability, followed by Pure Cotton with $\sim 20$ $\mathrm{g} / \mathrm{m} 2$. The strength properties are in dependence to the nature type of the fiber material and to the variety of the fiber content of the paper - more cotton fibers means enhance strength while bigger fiber variety ensures closed and evenly formed paper structure and fiber distribution. From the optical properties is seen that paper Blizzard and Organdie are more or less the same and they could be used for a similar labelling purposes. 
Table 1: Investigated characteristics of the studied papers

\begin{tabular}{|c|c|c|c|c|c|c|c|c|c|c|c|c|c|c|c|c|}
\hline \multirow[b]{2}{*}{ Properties } & \multicolumn{16}{|c|}{ Investigated papers } \\
\hline & $\begin{array}{l}\text { Dimensi } \\
\text { on }\end{array}$ & \multicolumn{3}{|c|}{$\begin{array}{l}\text { 1. Pure } \\
\text { Cotton }\end{array}$} & \multicolumn{3}{|c|}{ 2.Symphonie } & \multicolumn{3}{|c|}{ 3. Blizzard } & \multicolumn{3}{|c|}{ 4. Organdie } & \multicolumn{3}{|c|}{$\begin{array}{l}\text { 5. Velin } \\
\text { Caviar }\end{array}$} \\
\hline \multicolumn{17}{|c|}{ Structural and dimensional properties } \\
\hline I.1. Basic weight & $\mathrm{g} / \mathrm{m}^{2}$ & \multicolumn{3}{|c|}{100} & \multicolumn{3}{|c|}{90} & \multicolumn{3}{|c|}{90} & \multicolumn{3}{|c|}{95} & \multicolumn{3}{|c|}{90} \\
\hline I.2. Thickness & $\mathrm{mm}$ & \multicolumn{3}{|c|}{0,16} & \multicolumn{3}{|c|}{0,14} & \multicolumn{3}{|c|}{0,1} & \multicolumn{3}{|c|}{0,14} & \multicolumn{3}{|c|}{0,09} \\
\hline I.3. Density & $\mathrm{kg} / \mathrm{m}^{3}$ & \multicolumn{3}{|c|}{625} & \multicolumn{3}{|c|}{643} & \multicolumn{3}{|c|}{900} & \multicolumn{3}{|c|}{864} & \multicolumn{3}{|c|}{1000} \\
\hline I.4. Porosity & $\mathrm{m}^{3} / \mathrm{kg}$ & \multicolumn{3}{|c|}{0,0016} & \multicolumn{3}{|c|}{0,0015} & \multicolumn{3}{|c|}{0,0011} & \multicolumn{3}{|c|}{0,0012} & & 0,001 & \\
\hline Porosity & $\%$ & & 58,33 & & & 57,13 & & & 40 & & & 42,4 & & & 33,33 & \\
\hline $\begin{array}{l}\text { l.5. Smoothness } \\
\text { (Bekk), TS (top } \\
\text { side) }\end{array}$ & & & 1,4 & & & 2,06 & & & 24,93 & & & 3,55 & & & 18 & \\
\hline $\begin{array}{l}\text { Smoothness } \\
\text { (Bekk), BS (bottom } \\
\text { side) }\end{array}$ & S & & 1,35 & & & 1,52 & & & 24,33 & & & 3,22 & & & 18,33 & \\
\hline Hygrosco & c properti & & & & & & & & & & & & & & & \\
\hline $\begin{array}{l}\text { II.1. Water } \\
\text { absorption Cobb } 60 \text {, } \\
\text { TS }\end{array}$ & $a^{2}$ & & 19,67 & & & 17,28 & & & 21,95 & & & 16,42 & & & 25,9 & \\
\hline $\begin{array}{l}\quad \text { Water } \\
\text { absorption Cobb } 60 \text {, } \\
\text { BS }\end{array}$ & $\mathrm{g} / \mathrm{m}^{2}$ & & 20,68 & & & 18,56 & & & 20,92 & & & 16,79 & & & 25,25 & \\
\hline III. Strength & operties & & & & & & & & & & & & & & & \\
\hline $\begin{array}{l}\text { III.1. Tensile Index, } \\
\text { MD (machine } \\
\text { direction) }\end{array}$ & $\mathrm{N} m / \sigma$ & & 38,17 & & & 64,44 & & & 50,37 & & & 60,53 & & & 59,81 & \\
\hline $\begin{array}{l}\text { Tensile Index, } \\
\mathrm{CD} \text { (cross } \\
\text { direction) }\end{array}$ & N.m/g & & 25,17 & & & 41,48 & & & 26,17 & & & 30,88 & & & 35,56 & \\
\hline III.2. Burst Index & $\begin{array}{c}\mathrm{kPa} \cdot \mathrm{m}^{2} / \\
\mathrm{g}\end{array}$ & & 180 & & & 267 & & & 222 & & & 268 & & & 258 & \\
\hline $\begin{array}{l}\text { III.3. Tear Index, } \\
\text { MD }\end{array}$ & mN.m²/ & & 5,8 & & & 6 & & & 6,89 & & & 5,47 & & & 5,78 & \\
\hline $\begin{array}{ll} & \text { Tear Index, } \\
C D & \end{array}$ & & & 6,2 & & & 6,44 & & & 6,89 & & & 6,32 & & & 5,78 & \\
\hline Optical pr & perties & & & & & & & & & & & & & & & \\
\hline $\begin{array}{l}\text { IV.1. Whiteness } \\
\text { CIE }\end{array}$ & $\%$ & & 132,97 & & & 63,51 & & & 145,29 & & & 143,03 & & & $-0,60$ & \\
\hline $\begin{array}{l}\text { Whiteness } \\
\text { CIE - UV }\end{array}$ & $\%$ & & 75,13 & & & 62,35 & & & 87,64 & & & 90,95 & & & 0,09 & \\
\hline $\begin{array}{l}\text { IV.2. ISO } \\
\text { Brightness }\end{array}$ & $\%$ & & 95,45 & & & 78,30 & & & 93,75 & & & 94,25 & & & 4,02 & \\
\hline $\begin{array}{c}\text { ISO } \\
\text { Brightness - UV }\end{array}$ & $\%$ & & 85,29 & & & 78,16 & & & 83,68 & & & 85,26 & & & 4,04 & \\
\hline $\begin{array}{l}\text { IV.3. Fluorescense } \\
\text { CIE }\end{array}$ & & & 57,85 & & & 1,16 & & & 57,65 & & & 52,08 & & & $-0,69$ & \\
\hline $\begin{array}{l}\text { Fluoresense } \\
\text { ISO Brightness }\end{array}$ & $\%$ & & 10,15 & & & 0,14 & & & 10,07 & & & 8,98 & & & $-0,02$ & \\
\hline IV.4. Color coordina & & $\mathrm{L}^{*}$ & $a^{*}$ & $b^{*}$ & $\mathrm{~L}^{*}$ & $a^{*}$ & $b^{*}$ & $L^{*}$ & $a^{*}$ & $b^{*}$ & $\mathrm{~L}^{*}$ & $a^{*}$ & $b^{*}$ & $L^{*}$ & $a^{*}$ & $b^{*}$ \\
\hline & D65_10 & ตै & $\stackrel{n}{\stackrel{n}{\sim}}$ & $\frac{m}{\stackrel{m}{~}}$ & $\stackrel{m}{n}$ & 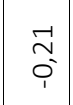 & $\stackrel{\infty}{\underset{\sigma}{\sim}}$ & 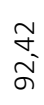 & 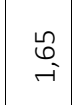 & $\begin{array}{l}\text { g } \\
\stackrel{-}{n} \\
\rightarrow\end{array}$ & $\begin{array}{l}\hat{0} \\
\text { n் }\end{array}$ & $\begin{array}{l}\infty \\
\infty \\
-i\end{array}$ & $\stackrel{\infty}{\underset{1}{N}}$ & 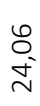 & $\hat{m}$ & 光 \\
\hline
\end{tabular}




\subsection{Investigation on the printability of the papers}

Pure Cotton is suitable for full colour offset printing, featuring that the colour density provided by the technology itself is not maximum. This is mainly due to the fact that one of the studied properties, namely the water absorption capacity of Cobb60, which for Pure Cotton is about $20 \mathrm{~g} / \mathrm{m}^{2}$, which is slightly below the optimal values of the offset printing index. It also has an exceptional effect on the reproduction of elements and strokes with screen printing. The absorbency of this paper combined with its porosity is so great that in fact highly pigmented inks, such as silver and gold bronze, are almost absorbed in depth and are poorly reproduced.

This paper has an exceptional pleasant to be touched texture, which is actually evident from the measurement of its smoothness (the lowest smoothness of all tested papers) considering its basic weight $-100 \mathrm{~g} / \mathrm{m}^{2}$. This quality makes it suitable for wine labels with clean designs. At first glance, the paper itself gives elegance and luxury to wine labels. And this is what attracts most designers and their desire to work with it.

From the samples made, it was found that the perception of the Hot foil is excellent, even the thin jewellery inscriptions and serifs are reproduced with high quality. The newly introduced technology in the world of Silk foil labels reproduces very well, behaved stable throughout printing.

From the printed samples was found out that at minimal pressure of the working section, the paper is easily torn, even the substrate is slightly cracked. This makes the paper not very suitable for embossing technology, not only for the production in Rotoprint, but also for many other productions, which is proved by the strength properties, showing lower values of tensile, tear and burst resistance. The quality of embossing could be judged by other indicators, such as porosity. Pure Cotton is the most porous of all the papers investigated - 58\%, which in combination with its weak strength properties can lead to problems in embossing irregularly shaped labels.

As a result of the research carried out, in Rotoprint two exemplary design of wine labels were created, which were produced with the printing and finishing techniques and technologies in accordance with the research and the end products are shown on Figure 7.

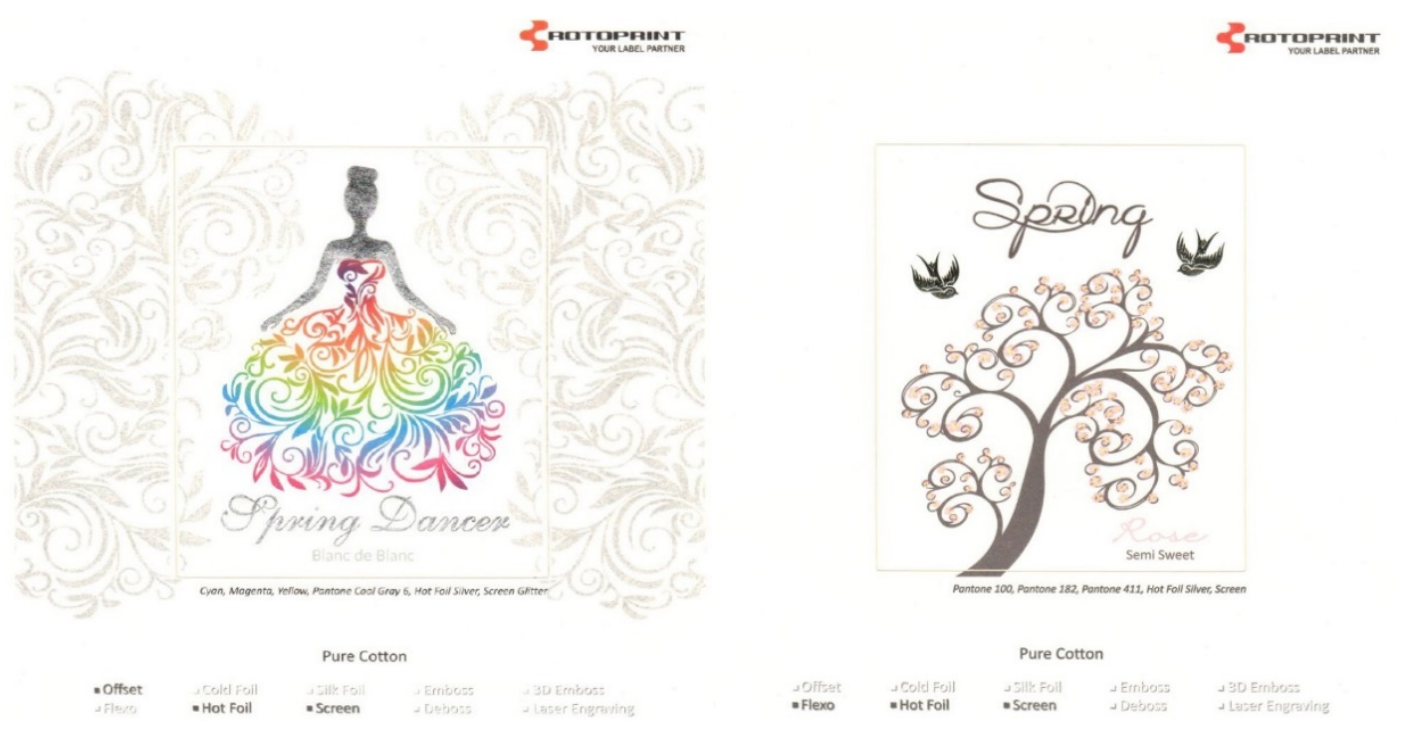

Figure 7: New designed labels on Pure Cotton paper

Excellent reproduction of the full-colour offset printing was established from the printed samples made on Symphonie paper. When printing on this paper, the colour density is higher, which might be due to its slight yellowness, but mostly is proven both visually and by measuring the optical properties of the tested papers (see Table 1). The established water absorption capacity of this type of paper is not very high compared to other papers, about $18 \mathrm{~g} / \mathrm{m}^{2}$ and the inks manage to fix on its surface very well.

A disadvantage is the reproduction of slightly torn and sometimes slightly jagged strokes in screen printing, which is largely due to the roughness of the paper - smoothness of the top side about $2 \mathrm{~s}$ was measured. The reproduction of very thin elements with the Silk and Hot foil technologies is very good, but there is a difference in the dense areas, where the texture of the material can be seen. This his is not always negative, in fact it can actually be a desired effect by a designer. 
Upon careful evaluation of the printed and converted samples we concluded that with this type of paper, the embossing is of an excellent quality, which is predictable from the results of the examined parameters, noted in the above table, due to its high porosity - $57 \%$ and very good strength properties. It is well known that when the porosity of the paper is high and is with higher plasticity it is a prerequisite for excellent embossing. Symphonie paper also has the highest values of resistance of tensile and burst, which is due not only to the high-quality fibrous material from which the paper is made (bleached cellulose from hard and softwood), but also to its excellent structural distribution, given by the fiber ratio (60:40\%) and its ability to form evenly distributed hydrogen bonds, even thou the paper is anisotropic, responsible for the strength of the paper. This is extremely important not only for the embossing technology, but also for the embossing of irregularly shaped labels, which produce a cutout of different width and shape. The produced designed wine labels on Symphonie paper, based on the research carried out are shown on Figure 8.

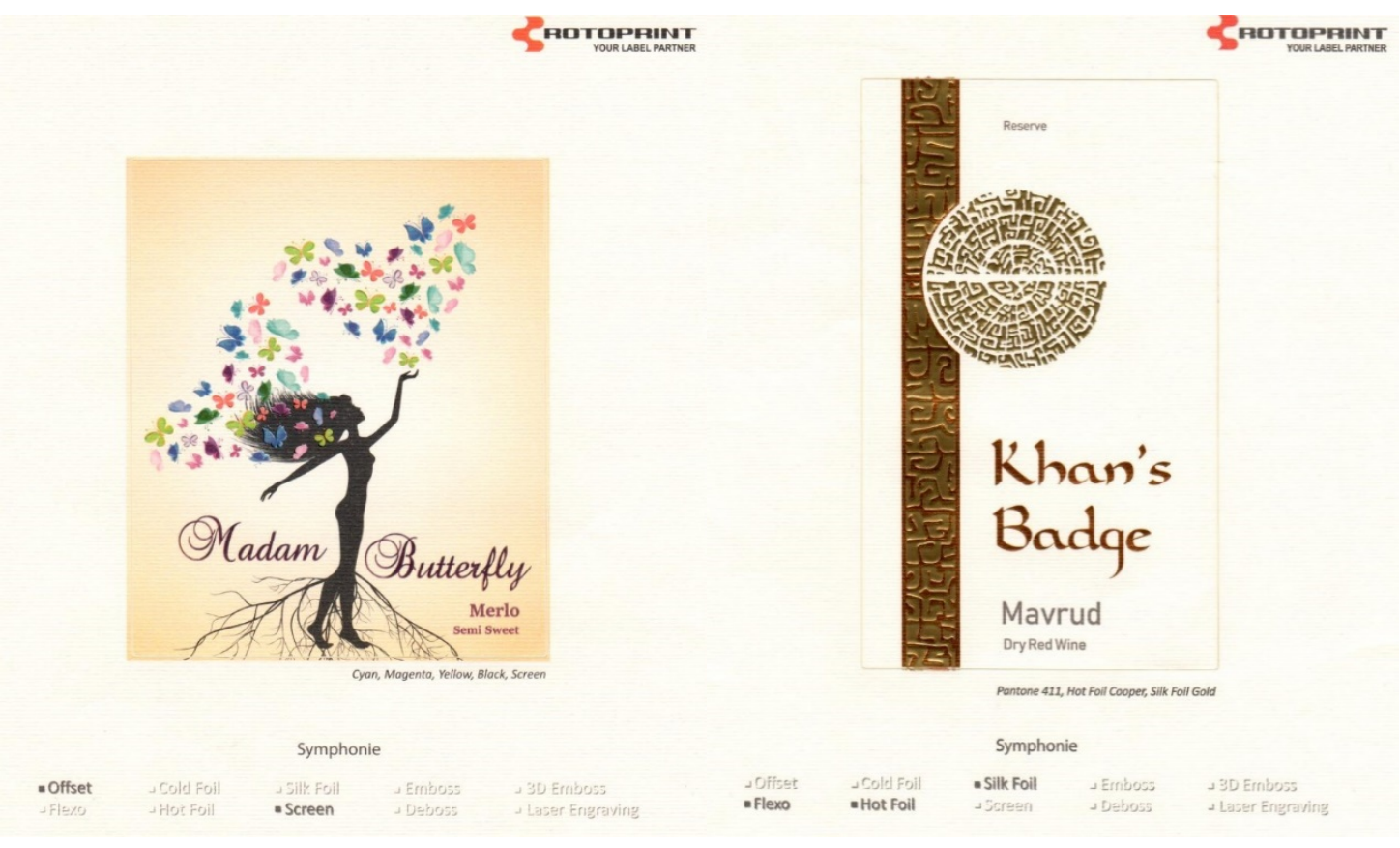

Figure 8: New designed labels on Symphonie paper

At first glance, Blizzard is a typical offset paper, with fine-feeling surface touch, with a very good printability, which makes it widely used in the label industry. This paper is preferred not only by wine label producers, but also for many other products in the food industry. From the printed samples the exceptional quality of the full-colour offset and flexo printing was established, with high optical density and well-fixed colours, even overflow and very good screen printing. Reproduction of thin, even jewellery elements, as well as dense areas with Silk foil and Hot stamping technologies are flawless. All these properties are due to the excellent combination of properties that this paper has, due to the variety of fibrous materials in its composition and the uniform structure of the paper sheet, both on the surface and in the core. Blizzard is characterized by high smoothness - about $25 \mathrm{~s}$ and compared to other types of paper studied, with the highest whiteness, optimal strength and hydrophobicity, medium to low porosity and relatively high density.

It is noteworthy that it has the highest tear resistance $-6.9 \mathrm{mN} . \mathrm{m}^{2} / \mathrm{g}$., and it depends primarily on the strength and nature of the fibers in the paper, but also from the structure. This is evidenced by microscopic analysis, which confirms the content of cotton fibers and spruce fibers in combination with two types of hardwood cellulose, which makes this paper with a closed structure, suitable for almost all printing and finishing processes. The produced wine labels on Blizzard paper, based on the research carried out are shown on Figure 9. 


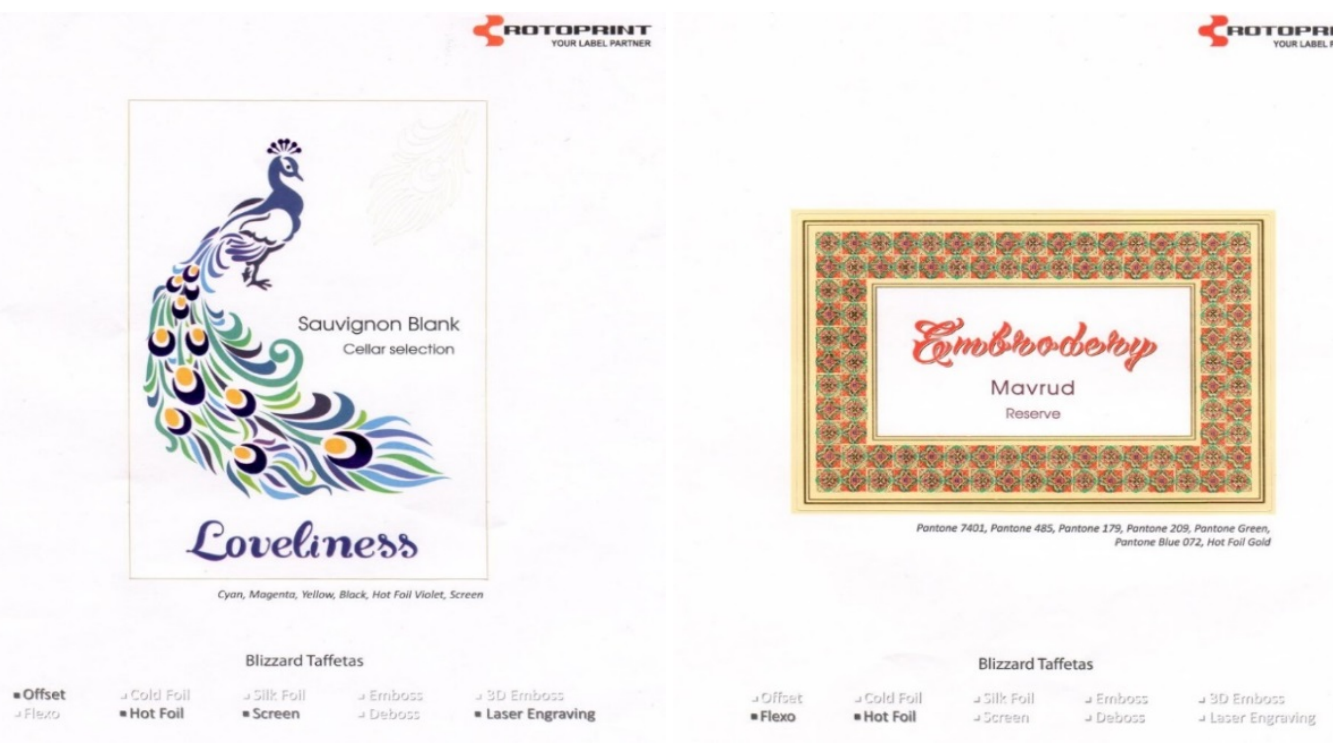

Figure 9: New designed labels on Blizzard paper

Organdie paper differs from the other studied papers with a special rough surface structure (resembling orange peel), characterized by low smoothness, about 3s, but on the other hand with good strength properties. This uneven surface has a negative effect on the printing of dense and very fine areas. Although it does not have a high water absorption capacity - about $16.5 \mathrm{~g} / \mathrm{m}^{2}$, the printing of strokes with screen printing technic is uneven, there is no sharpness of the elements. A similar problem occurs with dense areas with hot stamping. There is a "lintting" of the foil elements as a result of micro-irregularities on the surface of the paper. Also preferred for Silk Foil technology are elements of medium size, because the very thin ones are torn. In general, the reproduced colours are with high density due to the optical properties of Organdie - the paper has a high whiteness, thanks to the optical brightener. The samples made in Rotoprint show that the emboss is optimal due to the good physical and mechanical properties of the paper, despite the low porosity. Organdie paper could be widely used in the label industry, as it is suitable for clean designs, those that involve mainly finishing rather than printing processes. The produced wine labels on Organdie paper, based on the research carried out are shown on Figure 10.

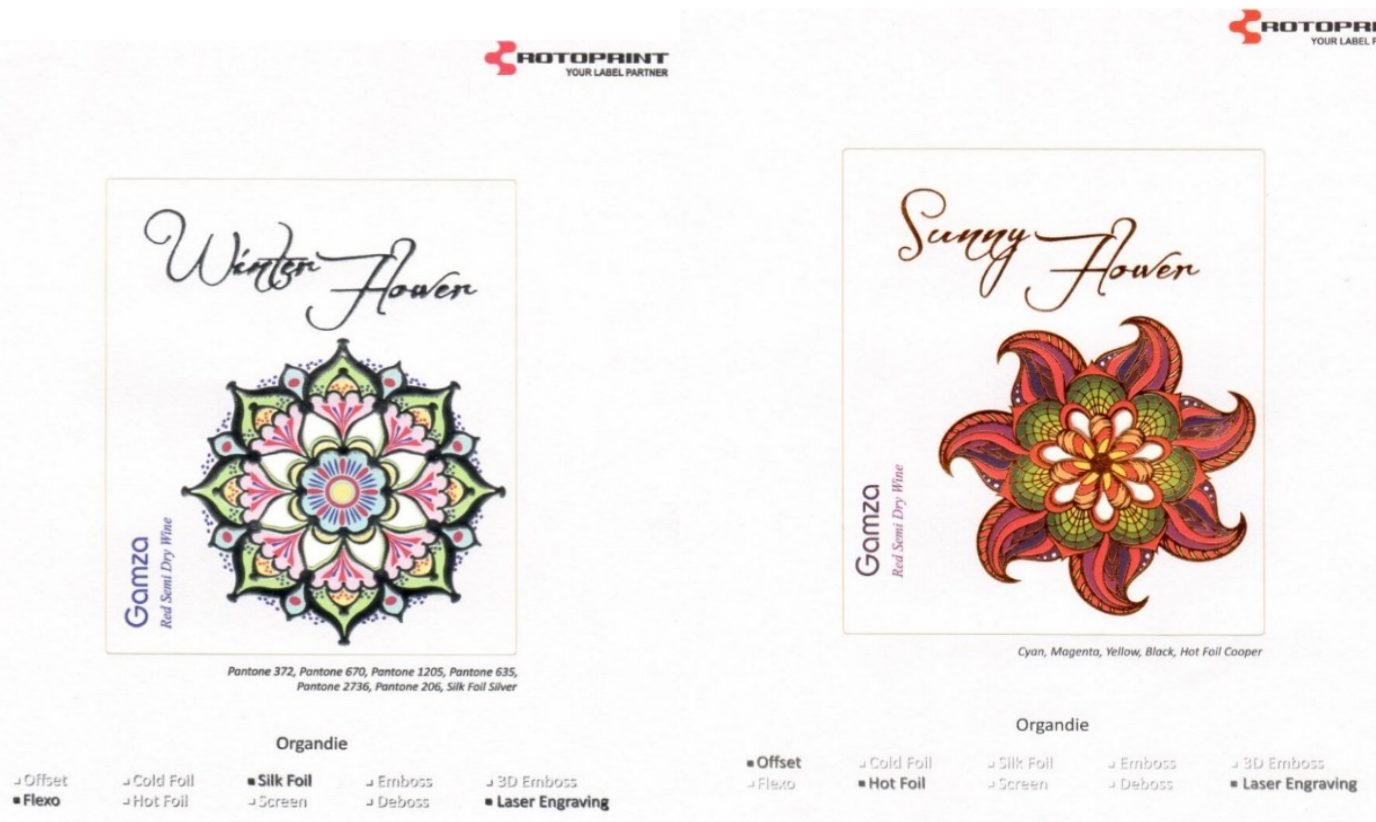

Figure 10: New designed labels on Organdie paper 
Velin Caviar paper differs significantly in colour from all the papers examined so far - it is coloured in suspension in deep black, which was established by microscopic analysis, and subsequently by engraving and laser perforation. The measured whiteness is as low as possible - ISO Brightness - 4.02\%.

This paper, like Blizzard, is attractive to designers because of its high smoothness and the velvety feeling of material when touched. It is characterized by the highest density of the investigated papers $-1000 \mathrm{~kg} / \mathrm{m}^{3}$, and has relatively good strength together with a good embossing. These properties are as a result of the balanced fibrous material composition of the paper, including cotton fibers and spruce wood fibers.

From the printed samples made it is clear that this paper is not suitable for full colour printing. Its absorbency is so high (just over $25 \mathrm{~g} / \mathrm{m}^{2}$ ) that even highly coating inks such as screen inks absorb into it. The only seal that is obtained is with highly pigmented gold and silver bronze, as well as screen varnish with glitter particles. All other processes such as Silk foil and Hot stamping and finishing are reproduced in high quality.

For this reason, the application of this paper is more limited and label designs could rely mainly on finishing processes. The produced designed wine labels on Velin Caviar paper, based on the research carried out are shown on Figure 11.

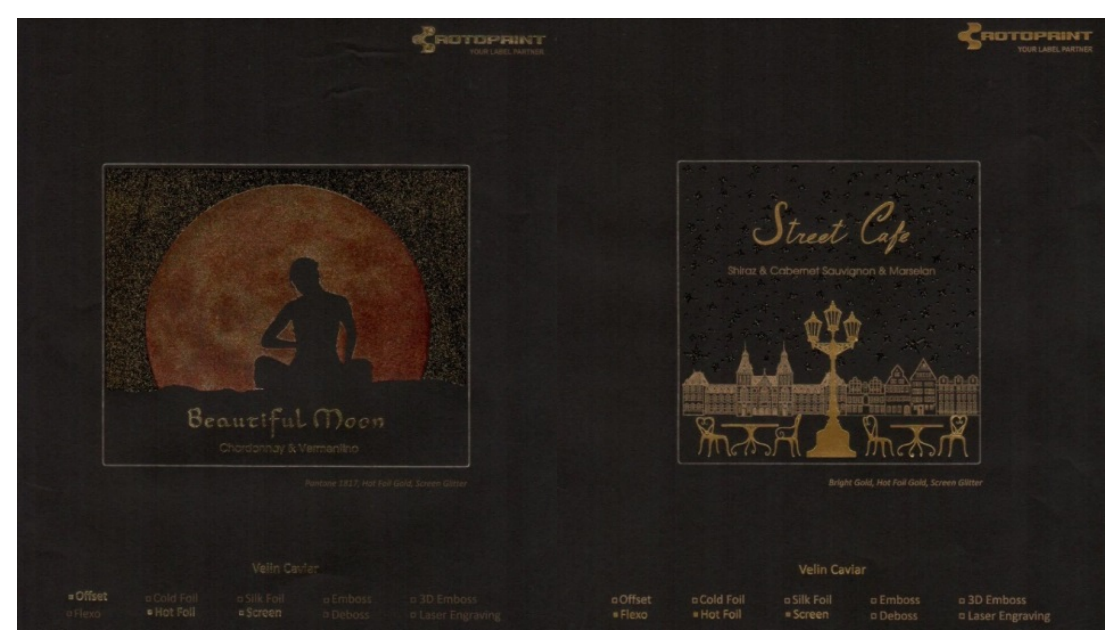

Figure 11: New designed labels on Velin Caviar paper

\section{CONCLUSION}

The conclusions of this scientific work could be summarized as follows:

- Paper from $100 \%$ natural cotton cellulose with high porosity and low strength properties could be used for the production of wine labels with clear designs through various printing techniques without finishing processes.

- Paper from 100\% natural bleached cellulose from softwood and hardwood species with high porosity and strength could be suitable for the production of wine labels through various printing techniques with variety of finishing processes.

- Paper with various fiber composition, including more than three wood materials, also cotton fibers with high density, low porosity and middle water absorption properties could be suitable for the production of wine labels through almost all printing and finishing processes.

- Paper with various fiber composition, including more than three wood materials, also cotton fibers with high density, low porosity and water absorption properties could be suitable for the production of wine labels with clean designs that involve mainly finishing rather than printing processes.

- Deeply dyed paper with various fiber composition, including more than three wood materials, also cotton fibers with very high density, low porosity and high water absorption capacity could be suitable for the production of wine labels that involve mainly finishing rather than printing processes.

- $\quad$ Papers with highly developed macrostructure of the surface (produced through marking felt) are characterized with a risk for dusting and lintting through the printing and finishing processes. 


\section{REFERENCES}

[1] EUR Lex: “Етикетиране на храните”, URL:

http://europa.eu/legislation_summaries/consumers/product_labelling_and_packaging/co0019_bg.h tm (last request: 2015-11-23).

[2] Franson, P.: "Labels Gone Wild", URL: https://www.winemag.com/2006/03/01/labels-gone-wild/ (last request: 2017-12-24).

[3] Holik, H.: "Handbook of Paper and Board", (Wiley-VCH, Weinheim, 2006.)

[4] Ivanova, N., Bencheva, S., Todorova, D.: "Handbook for exercises in chemistry, technology and properties of paper", (UCTM, Sofia, 2009.)

[5] Labels \& Labeling: "Printability testing of digitally printed labels", URL:

https://www.labelsandlabeling.com/news/latest/printability-testing-digitally-printed-labels (last request: 2010-07-07).

[6] Obis, E.: "Wine labels and consumer culture in the United States", InMedia:The French Journal of Media Studies, 2018. doi:https://doi.org/10.4000/inmedia.1029.

[7] Thorn, I., On Au, C.: „Applications of Wet-End Paper Chemistry”, (Springer, Berlin, 2009.)

[8] UPM Raflatac: "Paper Label Materials", URL: https://www.upmraflatac.com/products-andservices/label-products/paper-labels/ (last request: 2020-09-22).

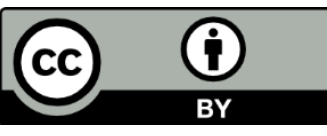

(C) 2020 Authors. Published by the University of Novi Sad, Faculty of Technical Sciences, Department of Graphic Engineering and Design. This article is an open access article distributed under the terms and conditions of the Creative Commons Attribution license 3.0 Serbia (http://creativecommons.org/licenses/by/3.0/rs/). 